Int. J. Advance Soft Compu. Appl, Vol. 13, No. 3, November 2021

Print ISSN: 2710-1274, Online ISSN: 2074-8523

Copyright (C) Al-Zaytoonah University of Jordan (ZUJ)

\title{
Recent Advances in Arabic Automatic Text
}

\section{Summarization}

\author{
Ahmad T. Al-Taani \\ Department of Computer Sciences, Yarmouk University, Irbid, Jordan \\ e-mail: ahmadta@yu.edu.jo
}

\begin{abstract}
Recently, the volume of the Arabic texts and documents on the internet had increased rabidly and generated a rich and valuable content on the $w w w$. Several parties had contributed to this content, this includes researchers, companies, governmental agencies, educational institutions, etc. With this big content it became difficult to search and extract useful information using only mankind skills and search engines. This motivated researchers to propose automated methodologies to extract summaries or useful information from those documents. A lot of research has been proposed for the automatic extraction of summaries for the English language and other languages. Unfortunately, the research for the Arabic automatic text summarization is still humble and needs more attention. This study presents a critical review and analysis of recent studies in Arabic automatic text summarization. The review includes all recent studies used the different text summarization approaches which include statistical-based, graph-based, evolutionary-based, and machine learning-based approaches. The selection criteria of the literature are based on the venue of publication and year of publication; back to five years. All review papers in Arabic ATS are excluded from the review since the study considers the recent methodologies in Arabic ATS. As a conclusion of this research, we recommend researchers in Arabic text summarization to investigate the use of machine learning on abstractive approach for text summarization due to the lack of research in this area.
\end{abstract}

Keywords: Automatic Text Summarization, The Arabic Language, Machine Learning, Natural Language Processing, Text Processing, Computational Linguistics. 


\section{Introduction}

Automatic text summarization (ATS) is one of the most important applications of NLP. ATS tools are very important for all kinds of people of different interests and education levels due to the rapidly increase of text data on the internet. As a result, researchers start spending efforts to enhance such applications. However, ATS tools help people to extract needed texts or documents faster and in a reasonable time. A Summary is a brief copy of the source document contains the most relevant text without redundancy and considering the coherence of the sentences in the summary.

Fig. 1 presents the most comprehensive criteria for classifying summaries. Lin \& Hovy [1] classified ATS systems according to several criteria. First criterion classifies ATS systems according to number of input documents, which could be single-document or multi-documents. In the case of single-document, the ATS system gives a summary of single-document, while for the multi-documents, the ATS systems generates a single summary for the multi-documents.

According to the second criterion, ATS systems are classified according to the method of summarization, abstractive and extractive. Abstractive methods generate a summary of the source document as human beings do; the summary is a shorter version of the source document written in different words. This method requires semantic analysis of the source document(s). Extractive methods generate a summary by extracting the most related sentences from the source document(s) according to some criteria such as the rank of the sentence and the summarization compression ratio [2].

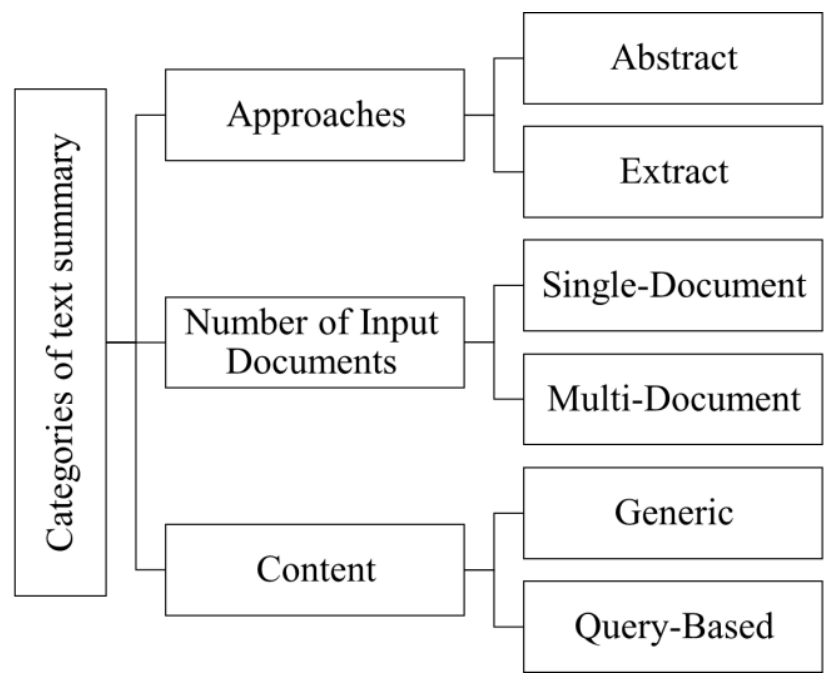

Fig. 1: The categories of text summary

The importance of the content of the source document is another criterion for classifying ATS systems this includes query-based summaries and generic summaries. In query-based systems, the user determines the topic of the source document as a query and then the system generates the summary that according to 
the user's query. Generic systems are used when the document subject is not considered, and the user does not understand the text [3].

ATS systems can be classified also according to the approaches used for generating the summary. The four main approaches for summarization are statistical approaches [4], graph-based approaches [5], machine learning approaches [6] and clustering-based approaches [7].

The rest of this paper is organized as follows: Section 2 presents Arabic ATS recent studies. This section in categorized into four main sub-sections according to the used approaches, these include statistical-based approaches, graph-based approaches, evolutionary-based approaches, and machine learning-based. Section 3 present the conclusion of this paper.

\section{Arabic ATS Recent Studies}

The evolution of Arabic applications and the increase of the Arabic speakers portrayed this language as one of the research topics on the ATS field. Although there are good efforts in the field of Arabic NLP, but unfortunately these efforts are not enough to cover this huge area. During the work on ATS, especially on Arabic language, different approaches have been proposed. Therefore, the aim of this study is to critically analyze the current approaches of Arabic ATS approaches. For each study, three elements are discussed, purpose of the study, research methodology, and findings. The review will also show the relationships between these approaches and will point out the similarities, differences, gaps, and weaknesses between these approaches.

The literature is categorized into four main categories according to the approaches used for Arabic ATS, these are: statistical-based approaches, graph-based approaches, evolutionary-based approaches, and machine learning-based approaches [8].

Although this study deals only with recent methodologies proposed for the Arabic ATS, there are several good review papers in this area. For instance, Ibrahim et al. [9] conducted a comparative study of Arabic multi-document ATS approaches. The review concluded that there is a lack of studies in Arabic ATS.

\subsection{Statistical-based approaches}

In these approaches, the summary is generated based on the ranking of the sentences by selecting the most relevant sentences from the source document(s) using suitable compression ratio. Relevant sentences are selected using significant features such as keywords, sentence length, sentence title, sentence position, term frequency (TF) and inverse-document frequency (IDF).

A statistical-based extractive approach for Arabic single-document summarization is suggested by Bialy et al [10]. The proposed approach comprised of three phases, 
a pre-processing, scoring of sentences, and summary generation. For the evaluation purpose, the authors collected 33 short articles (one to three paragraphs each) from the Wikipedia and summarized them by two human experts. Results are compared with the summaries of two human experts. The authors claimed good results compared to human experts' results.

Elayeb et al [11] presented an extractive approach for Arabic single-document summarization using analogical proportions by representing the relationship between documents and their corresponding summaries. Two algorithms are implemented, the first one checks for the existence of the keywords in the document or in the summary while the second algorithm considers the frequencies of the keywords as well. The two algorithms are compared with some summarizers like Luhn, TextRank, LexRank, and LSA using ANT corpus and a small test set from EASC. The two algorithms are evaluated using the ROUGE and BLEU metrics. Promising results are achieved compared with three other approaches using the same datasets.

Qaroush et al [12] proposed an extractive Arabic single-document summarization approach using supervised machine learning algorithms and a combination of statistical and semantic features. The EASC corpus and the ROUGE measure tool are used for the evaluation purpose. Good results are achieved compared to some previous approaches.

In [13], Ali proposed the use of LDA and modified PageRank to summarize Arabic and English documents. LDA algorithm is used to classify sentences as important or unimportant based on seven extracted features. Important sentences are used to construct the graph used by the modified PageRank algorithm to assign scores to each sentence in this graph. According to the authors, the proposed approach achieved promising results on the TAC dataset and the ROUGE metric tool.

Abdulateef et al [14] proposed the use of clustering and Word2Vec for Arabic multi-document text summarization. Vector space and bag-of-words models are used along with k-means clustering algorithm to reduce the redundancy of data for Arabic multi-documents. Preprocessing is applied first to remove noise from the data, then the words are converted into vectors using Word2Vec model, and the semantic relationships between vectors are represented. Following preprocessing, clustering is used for selecting the important sentences from the documents. The EASC dataset and the ROUGE metric are used for the evaluation purpose. Promising results are achieved compared to previous work in Arabic multidocument text summarization.

Table 1 summarizes the recent studies used statistical-based approaches. After a critical analysis of these studies, we conclude the following:

- All studies except [14] are applied on single documents.

- Studies in [11], [12], and [14] are used the same dataset (EASC). 
- All studies are evaluated their proposed approaches using the same metrics (precision, recall, and f-score).

- One study [13] used the TAC dataset.

- One study [10] used an artificial dataset consisting of 33 short documents collected from the Wikipedia. It is difficult to assess the significance of this study since it is not compared with other approaches and does not use a standard dataset as other studies. This study is also used a simple approach (TF-IDF) which is used by many researchers in the past.

- It can be seen from Table 1 that the study in [12] achieved better results on score-based approach compared to machine learning approaches. It is difficult to compare with the results of study [11] since different measures are used for evaluation.

Table 1: Summary of statistical-based approaches

\begin{tabular}{|c|c|c|c|c|c|}
\hline Ref. & Method & Dataset & Precision & Recall & F-score \\
\hline [10] & TF-IDF & $\begin{array}{c}33 \text { short } \\
\text { documents }\end{array}$ & 0.780 & 0.680 & 0.710 \\
\hline [11] & $\begin{array}{l}\text { analogical } \\
\text { proportions. }\end{array}$ & EASC & $\begin{array}{l}\text { ROUGE1 } \\
=0.75\end{array}$ & $\begin{array}{c}\text { BLEU1 } \\
=0.47\end{array}$ & - \\
\hline \multirow{2}{*}{ [12] } & $\begin{array}{l}\text { score-based } \\
\text { method }\end{array}$ & EASC & 0.616 & 0.673 & 0.643 \\
\hline & $\begin{array}{l}\text { machine } \\
\text { learning } \\
\text { algorithms }\end{array}$ & & 0.428 & 0.669 & 0.517 \\
\hline [13] & $\begin{array}{l}\text { LDA and } \\
\text { modified }\end{array}$ & TAC & 0.383 & 0.569 & 0.458 \\
\hline [14] & $\begin{array}{l}\text { PageRank. } \\
\text { k-means and } \\
\text { Word2Vec }\end{array}$ & EASC & 0.476 & 0.573 & 0.503 \\
\hline
\end{tabular}

\subsection{Graph-based approaches}

The focus of these approaches is on the semantic relationships between the document sentences. Sentences in the document are represented as a graph, nodes represent sentences, and the arcs represent relationship between sentences.

A graph-based approach for Arabic text summarization using modified PageRank algorithm is presented by Elbarougy et al [15]. The proposed approach consists of three phases: preprocessing phase, features extraction and graph construction phase, and summary extraction phase using the modified PageRank algorithm. The proposed approach achieved best results on the EASC dataset 10,000 iterations compared with other approaches. Best results achieved for precision, recall, and fmeasure are $0.687,0.729$, and 0.679 , respectively. 
Lamsiyah et al [16] proposed the use of transfer learning for Arabic multi-document text summarization. Syntactic and semantic relationships between words and phrases are captured by representing sentences using embedding vectors. Promising results are achieved compared with some previous work applied on three datasets: DUC2005, DUC2006, and DUC2007.

Elbarougy et al [17] investigated the effectiveness of morphological analysis using three morphological analyzers on the performance of graph-based approach for Arabic ATS. These analyzers are Safar Alkhalil, BAMA, and Stanford NLP. The EASC dataset is used to evaluate the performance of those morphological analyzers. According to this investigation, Safar Alkhalil analyzer achieved best results compared to other analyzers.

Elbarougy et al [18] investigated the impact of stop-words removal as a preprocessing step on the performance of graph-based approach for Arabic ATS. Two experiments are conducted, the first one, generating the summary without removing stop-words, and the second after removing stop-words. Experimental results performed on the EASC dataset showed that the performance of the summarization process gave better results when stop-words are removed.

Al-Taani and Al-Omour [19] investigated the effectiveness of using different basic units (word stem, the word itself, n-grams) on the performance of extractive graphbased approach for Arabic ATS. The proposed approach outperformed some of previous approaches when evaluated on the EASC dataset, and the ROUGE measure. Best results are achieved when n-grams are used in the summarization process.

Table 2 presents a summary of recent graph-based approaches reviewed in this paper. The summary is based on the methods used, the dataset or corpus, and the common measures used for evaluation. All approaches here are proposed for single document summarization except the approach in [16] is performed for multidocument summarization.

It can be inferred from Table 2 that the approach in [15] has achieved better results compared with the approaches in [17] and [18]. After a carful investigation of the proposed approaches by Elbarougy et al [17][18], we found the two approaches are similar with respect to the used methodology, i.e., graph-based approach, but they differ in the preprocessing step. In [17], three different morphological analyzers are investigated, and best results are achieved when Alkhalil analyzer is used. While in the second approach [18], two experiments are performed to investigate the effect of stop-words removal on the summarization process. Best results are achieved when experiments are conducted on the dataset without removing the stop-words. 
Table 2: Summary of graph-based approaches

\begin{tabular}{|c|c|c|c|c|c|}
\hline Ref. & Method & Dataset & Precision & Recall & F-score \\
\hline$[15]$ & $\begin{array}{l}\text { Modified } \\
\text { PageRank } \\
\text { algorithm }\end{array}$ & EASC & 0.687 & 0.729 & 0.679 \\
\hline \multirow[t]{4}{*}{ [16] } & TF-IDF, & $\begin{array}{l}\text { DUC2005 } \\
\text { DUC2006 }\end{array}$ & - & 0.408 & - \\
\hline & Word & DUC2007 & - & 0.439 & - \\
\hline & embeddings, & & - & 0.462 & - \\
\hline & $\begin{array}{c}\text { Sentence } \\
\text { embeddings. }\end{array}$ & & & & \\
\hline \multirow[t]{4}{*}{ [17] } & TF-IDF, Graphs, & EASC & Alkhalil: & 0.720 & 0.676 \\
\hline & Morphological & & 0.664 & 0.730 & 0.675 \\
\hline & analyzers & & $\begin{array}{l}\text { Stanford: } \\
0.655\end{array}$ & 0.714 & 0.671 \\
\hline & & & $\begin{array}{l}\text { BAMA: } \\
0.662\end{array}$ & & \\
\hline \multirow[t]{2}{*}{ [18] } & TF-IDF, Graphs & EASC & $\begin{array}{l}\text { with stop- } \\
\text { words: }\end{array}$ & 0.635 & 0.617 \\
\hline & & & $\begin{array}{c}0.602 \\
\text { without } \\
\text { stop-words: } \\
0.635\end{array}$ & 0.724 & 0.673 \\
\hline
\end{tabular}

\subsection{Evolutionary-based approaches}

Evolutionary-based approaches are heuristic-based approaches to solve problems such as combinatorial problems that cannot be easily solved in polynomial time e.g., NP-hard problems. Genetic algorithms are one example of evolutionary approaches.

An extractive approach for Arabic multi-document text summarization is proposed by Alqaisi et al [20]. This approach is based on clustering and multi-objective optimization algorithms. The two datasets TAC 2011 and DUC 2002 are used for the evaluation purpose. Results revealed the efficiency of the proposed approach compared to other existing approaches. The approach obtained good f-measure scores using the ROUGE metrics for both datasets.

Al-Abdallah \& Al-Taani [21] proposed the use of Particle swarm optimization (PSO) algorithm for Arabic single-document ATS. The EASC dataset and the ROUGE metric are used to evaluate the proposed model. Obtained results were promising compared to some existing approaches used genetic algorithms and Harmony search algorithm. 
Jaradat and Al-Taani [22] used genetic algorithms for Arabic single-document summarization. The results were compared with recent approaches used the EASC dataset and promising results were reported by the authors. These results are Recall $=0.5713$, Precision $=0.5658$, and F-measure $=0.5476$.

Table 3 shows a summary of evolutionary-based approaches. Unfortunately, only three recent studies were found in the literature, two of them [21] and [22] were applied of single documents, while the other study [20] is applied on multidocuments. The PSO approach [21] achieved better results compared to the GA approach in [22].

Table 3: Summary of evolutionary-based approaches

\begin{tabular}{cccccc}
\hline Ref. & Method & Dataset & Precision & Recall & F-score \\
\hline$[20]$ & $\begin{array}{c}\text { Clustering, } \\
\text { optimization }\end{array}$ & TAC 2011 & - & - & 0.389 \\
& DUC 2002 & 0.483 & 0.457 & 0.470 \\
{$[21]$} & PSO & EASC & 0.588 & 0.544 & 0.553 \\
{$[22]$} & $\begin{array}{c}\text { Genetic } \\
\text { algorithm }\end{array}$ & EASC & 0.565 & 0.571 & 0.547 \\
\hline
\end{tabular}

\subsection{Machine Learning-based approaches}

Machine learning and clustering approaches treat the summarization process as a classification problem, summary sentences are selected based on some features. Machine learning approaches are classified as supervised and unsupervised approaches. Examples of these approaches include Hidden Markov Models (HMM), and Bayesian approach. Clustering algorithms are used to represent sentence clusters, and then extract sentences from these clusters.

Lamsiyah et al [23] propose a deep learning approach for Arabic single document summarization using sentence embedding models. The DUC2002 dataset is used for the evaluation purpose using three sentence embedding models. Achieved results showed the effectiveness of the three sentence embedding models for Arabic text summarization in comparison with eight other approaches.

An Arabic dataset of Arabic summaries is created by Al-Maleh and Desouki [24]. The dataset consists of 300,000 entries consisting of an article introduction and the headlines for each introduction. Two abstractive models are proposed for the summarization of Arabic text using deep learning algorithms. Experimental results showed that the proposed models achieved good results using the created dataset. No comparisons are done with other approaches.

Suleiman \& Awajan [25] proposed the use of Recurrent Neural Network (RNN) for abstractive summarization of Arabic text. Two layers of hidden states at the encoder and one layer of hidden states at the decoder are used by the model. Long shortterm memory (LSTM) is used in the encoder and the decoder layers. The two layers of the encoder are the input text layer and the name entities layer. An artificial 
dataset is created and used for the evaluation of the summarization model using the ROUGE tool. Experimental results showed that the proposed model achieved good results for ROUGE1 and ROUGE1-NOORDER. Also, a comparison is made between the original word $2 \mathrm{Vec}$ model and the dependency-parsing-based word $2 \mathrm{Vec}$ model. Results revealed that dependency-parsing-based word2Vec model outperformed the original word $2 \mathrm{Vec}$ model.

Elgamal et al [26] investigated the effectiveness of different extractive text summarization approaches for Arabic text. These approaches include clustering algorithm with Latent Semantic Analysis (LSA), and deep learning approach using Deep Restricted Boltzmann Machine (DRBM). The proposed approaches are evaluated on an artificial dataset collected from Arabic educational book and Arabic stories. The experimental results showed that the deep learning approach (DRBM) achieved better results in comparison with the clustering-LSA approach.

Hummeida [27] proposed the use of Restricted Boltzmann Machine (RBM) for the extraction of summaries for the holy Quran interpretations. The proposed approach comprised of three phases: feature extraction, feature enhancement, and summary generation. First, features are extracted using RBM algorithm, then summaries for each single interpretation is generated using sentence scoring. The efficiency of the proposed approach is evaluated using precision and recall measures and achieved a precision of $0.49 \%$ and a recall of 0.46 .

A summary of machine learning-based approaches is presented in Table 4. Two studies [24] and [25] are used abstractive text summarization approach, while the other two studies [23] and [26] are used extractive approach. It is difficult to compare the performance of these studies since different artificial datasets and evaluation measured are used for the evaluation.

Table 4: Summary of machine learning-based approaches

\begin{tabular}{lccccc}
\hline Ref. & Method & Dataset & Precision & Recall & F-score \\
\hline$[23]$ & Neural Networks & DUC2002 & 0.621 & 0.344 & 0.442 \\
{$[24]$} & Deep learning & Artificial & 0.620 & 0.335 & 0.434 \\
& & & ROUGE1: 0.384 & \\
[25] & Deep learning. & Artificial & ROUGE1-NOORDER: 0.464 \\
& & & 0.49 & 0.45 & - \\
{$[27]$} & RBM & Holy & Quran & & \\
\hline
\end{tabular}

\section{Conclusion}

In this paper, a critical analysis of recent research in Arabic ATS is conducted. The three elements that mentioned for each study are: purpose of the study, research methodology, and findings. Most of the studies that used statistical-based approaches are performed on single documents and used extractive approach for summarization and used almost the same features as the TF-IDF. 
The achieved results were very close for all studies which made it very difficult to show the advantage of these studies over each other. Most of the studies that used graph-based approaches used the same steps as preprocessing, graph construction and the similarity measure. Some researchers investigated the effect of some preprocessing steps like the tool used in the morphological analysis and the necessity of stop-words removal step, but the results were very close which means that the preprocessing did not have a notable impact on the final summary. Unfortunately, little work has been done on the Arabic ATS using evolutionary approaches while there is a big interest on using machine learning-based approaches. In general, there is a lack of tools and datasets (corpora) for the purpose of Arabic ATS compared to the tools available for the other languages, this should be considered by researchers in the Arabic language to develop such tools and datasets. Also, most of the research in Arabic ATS is concentrated on the extractive approach of text summarization while the abstractive approach is very important since it emulates the human approach of summarization considering the sematic similarity of summaries. As a direction to future work, we encourage Arabic researchers to concentrate on the abstractive approach for Arabic ATS using deep learning algorithms. Another recommendation is to give intensive attention to corpora (datasets) creation due to the limitation of these resources for the Arabic language.

\section{References}

[1] Lin, C. Y., Hovy, E. (2002). From single to multi-document summarization: A prototype system and its evaluation, Proceedings of the 40th Annual Meeting on Association for Computational Linguistics. 2002 (pp.457-464).

[2] Radev, D. R., Hovy, E., McKeown, K. (2002). Introduction to the special issue on summarization, Computational linguistics, 28(4), 399-408.

[3] Ježek, K., Steinberger, J. (2008). Automatic Text Summarization (The stateof-the-art 2007 and new challenges), Proceedings of Znalosti (pp. 1-12).

[4] Lloret, E., Balahur, A., Palomar, M., Montoyo, A. (2009). Towards building a competitive opinion summarization system: challenges and keys, Proceedings of Human Language Technologies: The 2009 Annual Conference of the North American Chapter of the Association for Computational Linguistics, Companion Volume: Student Research Workshop and Doctoral Consortium (pp. 72-77), Association for Computational Linguistics.

[5] Wan, X., Yang, J. (2006). Improved affinity graph based multi-document summarization, Proceedings of the human language technology conference of the NAACL, Companion volume: Short papers (pp. 181-184), Association for Computational Linguistics. 
[6] Shareghi, E., Hassanabadi, L. S. (2008). Text summarization with harmony search algorithm-based sentence extraction, Proceedings of the 5th international conference on soft computing as transdisciplinary science and technology (pp. 226-231), ACM.

[7] Radev, D. R., Jing, H., Styś, M., Tam, D. (2004). Centroid-based summarization of multiple documents, Information Processing and Management, 40(6), 919-938.

[8] Al-Taani, A. T. (2017). Automatic Text Summarization Approaches, In: proceedings of the 2017 International Conference on Infocom Technologies and Unmanned Systems (ICTUS'2017), 18-20 December 2017, Dubai, UAE.

[9] Ibrahim, M. N., Abu Maria Kh., and Jaber, K. M. (2017). A Comparative Study for Arabic Multi-Document Summarization Systems (AMD-SS). In: proceedings of the $8^{\text {th }}$ International Conference on Information Technology (ICIT), pp. 1013-1022.

[10] Asmaa A. Bialy, Marwa A. Gaheen, R. M. ElEraky, A. F. ElGamal and Ahmed A. Ewees (2020). Single Arabic Document Summarization Using Natural Language Processing Technique, In: Abd Elaziz M., Al-qaness M., Ewees A., Dahou A. (eds) Recent Advances in NLP: The Case of Arabic Language. Studies in Computational Intelligence, vol 874. Springer, Cham. https://doi.org/10.1007/978-3-030-34614-0_2.

[11] Elayeb, B., Chouigui, A., Bounhas, M., Ben Khiroun, O. (2020). Automatic Arabic Text Summarization Using Analogical Proportions, Cognitive Computing 12, 1043-1069, https://doi.org/10.1007/s12559-020-09748-y.

[12] Qaroush, A., Abu Farha, I., Ghanem, W., Washaha, M., Maali, E. (2019). An efficient single document Arabic text summarization using a combination of statistical and semantic features, Journal of King Saud University - Computer and Information Sciences, https://doi.org/10.1016/j.jksuci.2019.03.010.

[13] Ali, Z. H. (2019). Multilingual Text Summarization based on LDA and Modified PageRank, Iraqi Journal of Information Technology, 9(3), 130160.

[14] Abdulateef, S., Khan, N.A., Chen, B., Shang, X. (2020). Multidocument Arabic Text Summarization Based on Clustering and Word2Vec to Reduce Redundancy, Information, 11, 59, https://doi.org/10.3390/info11020059.

[15] Elbarougy R., Behery, G., El Khatib, A. (2020a). Extractive Arabic Text Summarization Using Modified PageRank Algorithm, Egyptian Informatics Journal, 21(2), 73-81.

[16] Lamsiyah, S., El Mahdaouy, A., Ouatik El Alaoui, S., Espinasse, B. (2021). Unsupervised query-focused multi-document summarization based on transfer learning from sentence embedding models, BM25 model, and 
maximal marginal relevance criterion, Journal of Ambient Intelligence and Humanized Computing, https://doi.org/10.1007/s12652-021-03165-1.

[17] Elbarougy, R., Behery, G, and El Khatib, A. (2020b). Graph-Based Extractive Arabic Text Summarization Using Multiple Morphological Analyzers, Journal of Information Science and Engineering, 36, pp. 347363.

[18] Elbarougy, R., Behery, G., and El Khatibm A. (2019). The Impact of Stop Words Processing for Improving Extractive Graph-Based Arabic Text Summarization, International Journal of Scientific \& Technology Research, 8(11), 2134-2139.

[19] Al-Taani, A. T. and Al-Omour, M. (2014). An Extractive Graph-based Arabic Text Summarization Approach, Proceedings of the International Arab Conference on Information Technology (ACIT2014), University of Nizwa, Oman, December 9-11, pp. 158-163.

[20] Alqaisi, R., Ghanem, W., and Qaroush, A. (2020). Extractive MultiDocument Arabic Text Summarization Using Evolutionary Multi-Objective Optimization With K-Medoid Clustering, IEEE Access, 8, pp. 228206228224, Doi: 10.1109/ACCESS.2020.3046494.

[21] Al-Abdallah, R. Z. and Al-Taani, A. T. (2017). Arabic Single-Document Text Summarization Using Particle Swarm Optimization Algorithm, Procedia Computer Science, 117, pp. 30-37, https://doi.org/10.1016/j.procs.2017.10.091.

[22] Jaradat, Y. A. and Al-Taani, A. T. (2016). Hybrid-based Arabic singledocument text summarization approach using genetic algorithm, the 7th International Conference on Information and Communication Systems (ICICS), pp. 85-91, Doi: 10.1109/IACS.2016.7476091.

[23] Lamsiyah S., El Mahdaouy A., El Alaoui S.O., Espinasse B. (2020). A Supervised Method for Extractive Single Document Summarization Based on Sentence Embeddings and Neural Networks, In: Ezziyyani M. (eds) Advanced Intelligent Systems for Sustainable Development (AI2SD'2019), AI2SD 2019. Advances in Intelligent Systems and Computing, 1105, Springer, Cham. https://doi.org/10.1007/978-3-030-36674-2_8.

[24] Al-Maleh, M., Desouki, S. (2020). Arabic text summarization using deep learning approach, Journal of Big Data, 109, https://doi.org/10.1186/s40537-020-00386-7.

[25] Suleiman, D. and Awajan, A. (2020). Deep Learning Based Abstractive Text Summarization: Approaches, Datasets, Evaluation Measures, and Challenges, Mathematical Problems in Engineering, https://doi.org/10.1155/2020/9365340. 
[26] Elgamal, M., Hamada, S., Aboelezz, R., and Abou-Kreisha, M. (2019). Better Results in Automatic Arabic Text Summarization System Using Deep Learning based RBM than by Using Clustering Algorithm based LSA, International Journal of Scientific \& Engineering Research, 10(8), 781786.

[27] Hummeida, N. S. (2018). Text Summarization of Holy Quran Interpretation in English Using Deep Learning Algorithm, MSc Thesis, Sudan University of Science and Technology, Sudan.

\section{Notes on contributor}

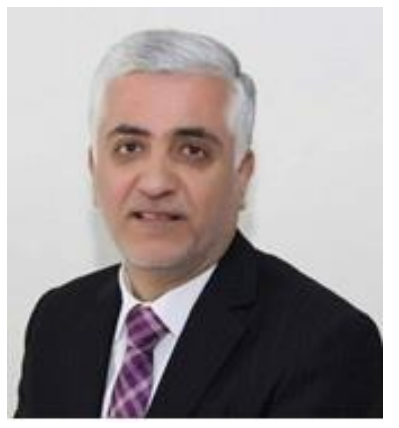

Ahmad T. Al-Taani is a full professor of Artificial Intelligence and a researcher at the Faculty of Information Technology and Computer Science, Department of Computer Science at Yarmouk university, Irbid, Jordan. He obtained his $\mathrm{PhD}$, MSc, and BSc degrees from university of Dundee (UK), National University (USA), and Yarmouk University (Jordan) in 1994, 1988 and 1985 respectively. Dr. Al-Taani has served on the Editorial Board for Journal of Pattern Recognition Research and a reviewer for several reputed journals including Natural Language Engineering, Soft Computing, Applied Soft Computing, Cognitive Computation, Cluster Computing, and others. His expertise areas include Arabic Natural Language Processing, Machine Learning, Knowledge-based Systems, Image Processing, Pattern Recognition, and Blockchain Technologies. 